(DOI: $10.24874 /$ jsscm.2017.11.01.02)

\title{
Implementing an Accurate Generalized Gaussian Quadrature Solution to Find the Elastic Field in a Homogeneous Anisotropic Media
}

\author{
H. Kabir ${ }^{1,}$ *, S. A. H. H. Matikolaei ${ }^{2}$ \\ ${ }^{1}$ Department of Civil and Mineral Engineering, University of Toronto, Toronto M5S 1A4, \\ Canada \\ e-mail: hossein.kabir@mail.utoronto.ca \\ ${ }^{2}$ Department of Civil and Environmental Engineering, Sharif University of Technology, Tehran \\ 145888-9694, Iran \\ e-mail: amirh.hassanpour@gmail.com \\ *corresponding author
}

\begin{abstract}
In the current study, the elastic field in an anisotropic elastic media is determined by implementing a general semi-analytical method. In this specific methodology, the displacement field is computed as a sum of finite functions with unknown coefficients. These aforementioned functions exactly satisfy both the homogeneous and inhomogeneous boundary conditions in the proposed media. It is worth mentioning that the unknown coefficients are determined by implementing the principle of minimum potential energy. The numerical integration is done by employing the Generalized Gaussian Quadrature rule. Furthermore, and with the aid of the calculated unknown coefficients, the displacement fields as well as the other parameters of the elastic field are obtainable. Finally, the comparison of the previous analytical method with the current semi-analytical approach proposes the efficacy of the present methodology.
\end{abstract}

Keywords: Anisotropic Elastic Media, Semi-Analytical Method, Elastic Field, Generalized Gaussian Quadrature Solution

\section{Introduction}

Determining the elastic field in an anisotropic material using an analytical method is always regarded as a time-consuming process, especially if the geometry of the assumed media is complex. In general, a proper Airy stress function proposed for an elastic or rigid media subjected to a specific loading (Gdoutos 2003, Kabir et al. 2011, Seif et al. 2015, Mitchell et al. 2016, Alshaya et al. 2017). In this specific method, the proposed function is generally dependent on the geometry of the assumed problem. In the literature, the minimum potential energy method could be implemented for composite materials using a semi-analytical method (Weertman et al. 1996, Kabir et al. 2010, Seif et al. 2016, Seif et al. 2017, Kabir et al. 2017). Where in these cases, the elastic field for an assumed media is considered under the applied mechanical loading. In addition, the minimum potential energy method for a cracked anisotropic media is employed using a semi-analytical method (Kabir 2005, Hojatkashani et al. 2012, Zarrinzadeh et al. 2016, Alaedini et al. 2016, Zarrinzadeh et al. 2017). To go further beyond that, the minimum potential energy method has been taken into consideration to find the stress concentration in elastostatics 
(Morley 1969, Lu et al. 2007). It is worth noting that the energy-based methods are presented to find the inter-laminar stresses in a layered anisotropic media (Kabir et al. 2007, Daouadji 2007, Eslami et al. 2015). And in a more recent times, many mesh-free methods are implemented to find the stress distribution along the assumed elastic or rigid media (Kabir 1997, Eynbeygi et al. 2014, Sahmani et al. 2017).

In the current study, the elastic field in an anisotropic media is investigated by implementing the principle of minimum potential energy. In this very approach, the displacement field could be computed as a sum of proposed finite functions with unknown coefficients by applying the Generalized Gaussian Quadrature rule as an integration method. By minimizing the system's total potential energy with respect to these unknown coefficients, the correspondent elastic field could be evaluated.

\section{Fundamental Relations}

Generally, the anisotropic elastic media with arbitrary orientation and boundary conditions could be demonstrated as Fig. 1.



Fig. 1. Anisotropic material with arbitrary loading and boundary condition

In addition, the total potential energy of this specific domain would be given as

$$
\Pi=\frac{1}{2} \iint_{\Omega} \varepsilon: \sigma d \Omega-\iint_{\Omega} \rho b \cdot u d \Omega-\iint_{\Gamma_{t}} t^{0} \cdot u d \Gamma
$$

where $\rho$ is the mass density, $b$ is the body force field, $t^{0}$ is the prescribed traction on an specific part of the boundary $\Gamma_{\mathrm{t}}$, and $u$ is the displacement field.

The stress-strain relation in the form of matrix notation for an anisotropic media in twodimensional elasticity could be expressed as

$$
\left(\begin{array}{l}
\sigma_{11} \\
\sigma_{22} \\
\sigma_{12}
\end{array}\right)=\left(\begin{array}{lll}
C_{11} & C_{12} & C_{16} \\
C_{12} & C_{22} & C_{26} \\
C_{16} & C_{26} & C_{66}
\end{array}\right)
$$

In Fig.1, the strain-displacement relations could be expressed as follows: 


$$
\begin{gathered}
\frac{\partial}{\partial x_{1}}=\frac{\partial r}{\partial x_{1}} \frac{\partial}{\partial r}+\frac{\partial \theta}{\partial x_{1}} \frac{\partial}{\partial \theta}, \frac{\partial}{\partial x_{2}}=\frac{\partial r}{\partial x_{2}} \frac{\partial}{\partial r}+\frac{\partial \theta}{\partial x_{2}} \frac{\partial}{\partial \theta} \\
\varepsilon_{11}=\frac{\partial u_{1}}{\partial x_{1}}=\frac{\partial r}{\partial x_{1}} \frac{\partial u_{1}}{\partial r}+\frac{\partial \theta}{\partial x_{1}} \frac{\partial u_{1}}{\partial \theta} \\
\varepsilon_{22}=\frac{\partial u_{2}}{\partial x_{2}}=\frac{\partial r}{\partial x_{2}} \frac{\partial u_{2}}{\partial r}+\frac{\partial \theta}{\partial x_{2}} \frac{\partial u_{2}}{\partial \theta} \\
2 \varepsilon_{12}=\frac{\partial u_{1}}{\partial x_{2}}+\frac{\partial u_{2}}{\partial x_{1}}=\frac{\partial r}{\partial x_{2}} \frac{\partial u_{1}}{\partial r}+\frac{\partial \theta}{\partial x_{2}} \frac{\partial u_{1}}{\partial \theta}+\frac{\partial r}{\partial x_{1}} \frac{\partial u_{2}}{\partial r}+\frac{\partial \theta}{\partial x_{1}} \frac{\partial u_{2}}{\partial \theta}
\end{gathered}
$$

Regarding Fig. 1, the Cartesian to polar transformations are expressed as:

$$
\frac{\partial r}{\partial x_{1}}=\cos \theta, \frac{\partial r}{\partial x_{2}}=\sin \theta, \frac{\partial \theta}{\partial x_{1}}=-\frac{\sin \theta}{r}, \frac{\partial \theta}{\partial x_{2}}=-\frac{\cos \theta}{r}
$$

In addition, by substituting Eq. (7) into Eqs. (3) to (6), the new strain-displacement relations could be derived and presented as follows:

$$
\begin{gathered}
\varepsilon_{11}=\frac{\partial u_{1}}{\partial x_{1}}=\cos \theta \frac{\partial u_{1}}{\partial r}-\frac{\sin \theta}{r} \frac{\partial u_{1}}{\partial \theta} \\
\varepsilon_{22}=\frac{\partial u_{2}}{\partial x_{2}}=\sin \theta \frac{\partial u_{2}}{\partial r}+\frac{\cos \theta}{r} \frac{\partial u_{1}}{\partial \theta} \\
2 \varepsilon_{12}=\frac{\partial u_{1}}{\partial x_{2}}+\frac{\partial u_{2}}{\partial x_{1}}=\sin \theta \frac{\partial u_{1}}{\partial r}+\frac{\cos \theta}{r} \frac{\partial u_{1}}{\partial \theta}+\cos \theta \frac{\partial u_{2}}{\partial r}-\frac{\sin \theta}{r} \frac{\partial u_{2}}{\partial \theta}
\end{gathered}
$$

The displacement field in the proposed anisotropic media could be written as a sum of polynomial finite series:

$$
\begin{gathered}
u_{i}=\left\{\sqrt{r} \sum_{m=1}^{n_{r}} \sum_{n=0}^{n_{0}}(r)^{m}\left[A_{m n}^{i} F_{n}(\theta)+B_{m n}^{i} G_{n}(\theta)\right]\right\}, i=1,2 \\
\text { where, } \\
F_{n}(\theta)=\sin (n+1) \theta, G_{n}(\theta)=\cos \frac{n}{2} \theta \quad-\pi \leq \theta \leq \pi
\end{gathered}
$$

In order to avoid the singularity in Eq. (11), the term "m" must be started from m=1, due to the fact that the stress singularity $\frac{1}{\sqrt{r}}$ corresponds to the very $m=0$ term, and in the current study, the assumed problem is continuous, therefore the $F_{n}(\theta)$ and $G_{n}(\theta)$ functions are defined on the interval of $-\pi \leq \theta \leq \pi$, where the definitions of $\mathrm{r}$ and $\theta$ terms are denotes in Fig. 1 as well. To minimize the system total potential energy with respect to the unknown coefficients, a system of linear equations could be derived as follows: 


$$
H X=Q
$$

where the unknown coefficient, that is X, could be expressed as in Eq. (14)

$$
X^{T}=\left\{B_{m n}^{i} A_{m n}^{i}\right\} i=1,2 . m=1,2, \ldots, n_{r} \cdot n=0,1,2, \ldots, n_{\theta}
$$

Henceforth, and with the aid of evaluated unknown coefficients, the displacement field and consequently the elastic field could be computed.

\section{Finding the Elastic Field in an Isotropic Rotating Shaft/Disc with an Arbitrary Oriented Central Elliptical Perforated Hole}

In this example, an elliptical rotating shaft/disc with an arbitrary central perforated elliptical hole is shown in Fig. 2. The $a_{1}$ and $b_{1}$ parameters are the ellipse major axes of the assumed disc in Fig. 3 , and the $a_{2}$ and $b_{2}$ parameters are the major axes of the perforated elliptical hole. The only force acting on this specific media is the rotational body force field, which is a centrifugal force.

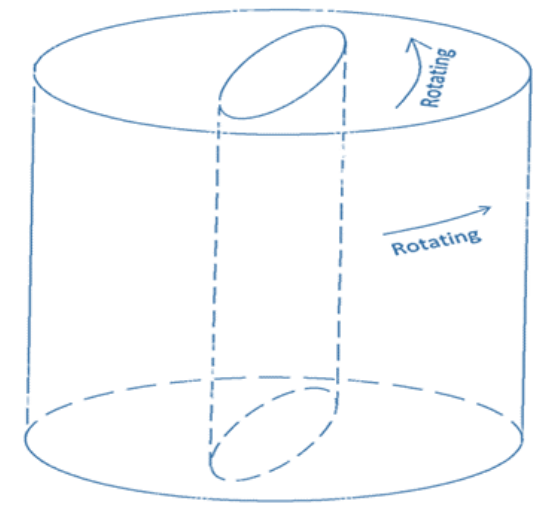

Fig. 2. Rotating cylindrical shaft



Fig. 3. Rotating shaft cross-section

The displacement fields proposed in Eq. (11) in perpendicular directions are assumed and selected as follows: 


$$
\begin{gathered}
u_{1}=\sqrt{r} \sum_{m=1}^{n_{r}} \sum_{n=0}^{n_{0}}(r)^{m}\left[B_{m n}^{i} \cos \frac{n}{2} \theta\right] \\
u_{2}=\sqrt{r} \sum_{m=1}^{n_{r}} \sum_{n=0}^{n_{0}}(r)^{m}\left[A_{m n}^{i} \sin (n+1) \theta\right]
\end{gathered}
$$

Regarding Eqs. (8) to (10), the strain-displacement relations could be derived as follows:

$$
\begin{gathered}
\varepsilon_{11}=\frac{\partial u_{1}}{\partial x_{1}}=\sum_{m=1}^{n_{r}} \sum_{n=0}^{n_{0}}(r)^{m-0.5}\left[B_{m n}^{i}\left(\frac{m+0.5}{a} \cos \frac{n}{2} \theta \cos \theta+\frac{n}{2 a} \sin \frac{n}{2} \theta \sin \theta\right)\right] \\
\varepsilon_{22}=\frac{\partial u_{2}}{\partial x_{2}}=\sum_{m=1}^{n_{r}} \sum_{n=0}^{n_{0}}(r)^{m-0.5}\left[A_{m n}^{i}\left(\frac{m+0.5}{a} \sin (n+1) \theta \sin \theta+\frac{n+1}{a} \cos (n+1) \theta \cos \theta\right)\right] \\
2 \varepsilon_{12}=\sum_{m=1}^{n_{r}} \sum_{n=0}^{n_{0}}(r)^{m-0.5}\left[B_{m n}^{i}\left(\frac{m+0.5}{a} \cos \frac{n}{2} \theta \sin \theta+\frac{n}{2 a} \sin \frac{n}{2} \theta \cos \theta\right)+\right. \\
\left.A_{m n}^{i}\left(\frac{m+0.5}{a} \sin (n+1) \theta \cos \theta-\frac{n+1}{a} \cos (n+1) \theta \sin \theta\right)\right]
\end{gathered}
$$

And for the elastic isotropic material, the stress-strain relation is expressed in Eq. (20).

$$
\left(\begin{array}{l}
\sigma_{11} \\
\sigma_{22} \\
\sigma_{12}
\end{array}\right)=\left(\begin{array}{ccc}
C_{11} & C_{12} & 0 \\
C_{12} & C_{22} & 0 \\
0 & 0 & C_{66}
\end{array}\right)\left(\begin{array}{l}
\varepsilon_{11} \\
\varepsilon_{22} \\
\varepsilon_{12}
\end{array}\right), C_{11}=C_{22}, C_{12}=C_{21}
$$

Thus, the system's total potential energy, in terms of strain energy and body force, in the absence of surface traction, is represented in Eq. (21).

$$
\Pi=\frac{1}{2} \iint \Omega \varepsilon: \sigma d \Omega-\iint_{\Omega} \rho b \cdot u d \Omega
$$

Integration over the assumed elliptical zone would be possible by discretizing the assumed domain into triangular sections, shown in Fig. 4. As a representation of this discretization, the disc is divided into 24 triangles. In each triangle, the Gaussian points are assigned and summed over the admissible area. Thus, for each area, each Gauss point has its own weight point, as represented in Fig. 5. 



Fig. 4. Dividing the disc domain into multiple triangular sections

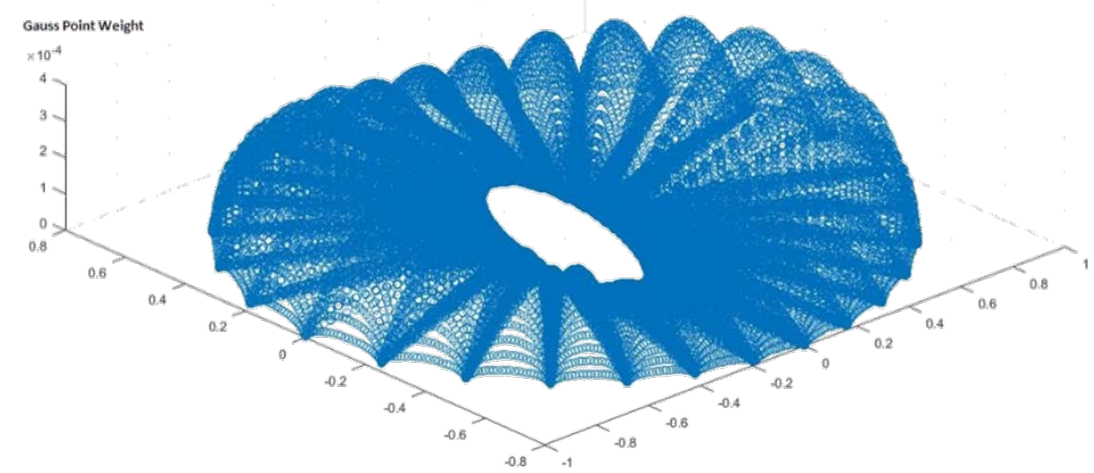

Fig. 5. Projection of the Gaussian weight point over the assumed disc media

Following the computed displacement field, Eq. (21) must be minimized and derived with respect to the unknown coefficients, that is, the components of X matrix in Eq. (14) in order to find the elastic field in that assumed media.

\section{Results Verifications: Circular Rotating Disc with a Perforated Circular Hole}

For this very condition, and regarding Fig. 3, the following assumptions were made (Table 1) with corresponding figure (Fig. 6):

\begin{tabular}{cc}
\hline$v=0.4$ (Poisson's ratio) & $a_{1}=b_{1}=1.0$ (Disc. major axes) \\
\hline$\theta=0^{\circ}$ (Hole orientation) & $a_{2}=b_{2}$ (Hole major axes) \\
\hline$\omega=1.0$ (Rotational velocity) & $n_{r}=n_{\theta}=10$ (Finite polynomial Series) \\
\hline
\end{tabular}

Table 1. Numerical assumptions for the results verification 


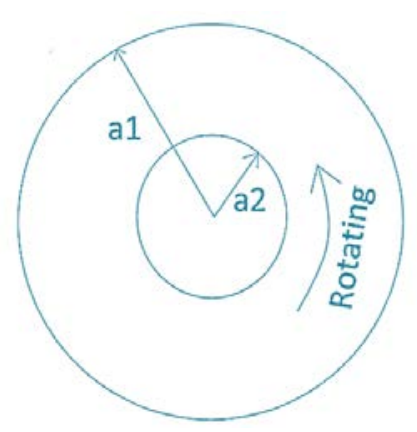

Fig. 6. Circular rotating disc with a perforated circular hole

The closed-form solution for the hoop stress of a rotating annular circular disc under the plain strain condition is computed as follows in Eq. (22), (Çetin et al. 2014)

$$
\sigma_{\theta \theta}=\rho \cdot \frac{\omega^{2}}{8} \cdot\left[(3+v)\left(\alpha_{1}^{2}+a_{2}^{2}+\frac{a_{1}^{2} \cdot a_{2}^{2}}{r^{2}}\right)-(1+3 v) \cdot r^{2}\right]
$$

By comparing the results of the both former analytical solution and the present semianalytical method, which are shown in Table 2 and Fig. 7, the accuracy of the proposed method is verified as well.

\begin{tabular}{ccc}
\hline $\mathrm{a}_{2}=\mathrm{b}_{2}$ (Hole Major & $\boldsymbol{\sigma}_{\boldsymbol{\theta} \boldsymbol{\theta}}$ Analytical (Exact) & $\boldsymbol{\sigma}_{\boldsymbol{\theta} \boldsymbol{\theta}}$ Semi-Analytical (Current Study) \\
\hline 0.15 & 0.2968 & 0.2887 \\
\hline 0.2 & 0.3175 & 0.3067 \\
\hline 0.25 & 0.3440 & 0.3371 \\
\hline 0.3 & 0.3765 & 0.3646 \\
\hline 0.35 & 0.4149 & 0.3921 \\
\hline 0.4 & 0.4592 & 0.4463 \\
\hline 0.45 & 0.5093 & 0.4945 \\
\hline 0.5 & 0.5654 & 0.5582 \\
\hline 0.55 & 0.6274 & 0.6122 \\
\hline 0.6 & 0.6953 & 0.7025 \\
\hline 0.65 & 0.7691 & 0.7686 \\
\hline
\end{tabular}

Table 2. Comparing the Hoop stress for both analytical and semi-analytical results 




Fig. 7. Comparison of the Hoop stress for both analytical and semi-analytical results

\section{Conclusion}

Based on the comparison made between the former analytical method and the current semianalytical approach, the results prove the accuracy of the proposed method. Furthermore, this mesh-less approach could be applied to any anisotropic media regardless of the complexity of its physical shape. Moreover, the present methodology could be applied to a cracked anisotropic or composite media with arbitrary crack orientations, where finding the traditional method of finding the analytical solution may be regarded as an impossible task to follow.

\section{Acknowledgment}

H. K would like to acknowledge partial supports from Sharif University of Technology, and the priceless guidance of Prof. H. M. Shodja, and Dr. M. T. Kamali, faculties of Sharif University of Technology and Hormozgan University, respectively.

\section{References}

Alaedini S, Mohammad Z, and Hadi H (2016). Seismic ductility evaluation of shear-deficient RC frames strengthened by externally bonded CFRP sheets. KSCE Journal of Civil Engineering 20, 5, 1925-1935.

Alshaya A, Shuai X, Rowlands R (2017). Stress Analysis of a Finite Orthotropic Plate Containing an Elliptical Hole from Recorded Temperature Data. In Residual Stress, Thermomechanics \& Infrared Imaging, Hybrid Techniques and Inverse Problems, Volume 9, Springer International Publishing, 47-56.

Çetin E, Kurşun A, Aksoy Ş. Tunay Çetin M (2014). Elastic Stress Analysis of Annular BiMaterial Discs with Variable Thickness under Mechanical and Thermomechanical Loads. World Academy of Science, Engineering and Technology, International Journal of Mechanical, Aerospace, Industrial, Mechatronic and Manufacturing Engineering 8, 2, 288292.

Daouadji TH (2017). Analytical and numerical modeling of interfacial stresses in beams bonded with a thin plate. Advances in Computational Design 2, 1, 57-69.

Eslami G and Kabir MZ (2015). Multi-objective optimization of orthogonally stiffened cylindrical shells using optimality criteria method. Scientia Iranica. Transaction A, Civil Engineering, 22(3), 717. 
Eynbeygi M and Aghdam MM (2015). A micromechanical study on the electro-elastic behavior of piezoelectric fibrous composites using element free Galerkin method. Modares Mechanical Engineering 14 (6), 175-184.

Gdoutos EE (2003). Airy Stress Function Method in Problems of Fracture Mechanics and Fatigue, Springer Netherlands, 3-9.

Hojatkashani A and Kabir MZ (2012). Interfacial stress assessment at the cracked zones in CFRP retrofitted RC beams, Structural Engineering and Mechanics 44, 6, 705-733.

Kabir MZ (1997). A Finite Element Presentation of Optimum Design for Filament-Winding Composite Cylindrical Pressure Vessels. Solid Mechanics and its Applications, 54, 95-104.

Kabir MZ (2005). Structural performance of 3-D sandwich panels under shear and flexural loading, Journal of Scientifica Iranica 12, 4, 402-408.

Kabir MZ and Hojatkashani A (2007). Analytical and Finite Element Study of Cracking and Interfacial Stress Distribution at the Cracked Zone in a Retrofitted Rc Beam.

Kabir MZ and Seif AE (2010). Lateral-torsional buckling of retrofitted steel I-beams using FRP sheets, Scientia Iranica. Transaction A, Civil Engineering 17, 4, 262.

Kabir MZ and Seif AE (2011). Lateral torsional buckling of steel i-beam retrofitted using FRP sheets: analytical solution and optimization, Advances in FRP Composites in Civil Engineering, Springer Berlin Heidelberg, 915-918.

Kabir H and Sadeghi M (2017). Unconfined Strength of Nano Reactive Silica Sand Powder Concrete, World Academy of Science, Engineering and Technology, International Journal of Civil, Environmental, Structural, Construction and Architectural Engineering 11, 3, 356360.

Lu J, Zhou X and Raghavan ML (2007). Inverse elastostatic stress analysis in pre-deformed biological structures: demonstration using abdominal aortic aneurysms, Journal of biomechanics 40, 3, 693-696.

Mitchell T, Baker W, McRobie A and Mazurek A (2016). Mechanisms and states of self-stress of planar trusses using graphic statics, part I: The fundamental theorem of linear algebra and the Airy stress function, International Journal of Space Structures 31, 2-4, 85-101.

Morley LSD (1969). A modification of the Rayleigh-Ritz method for stress concentration problems in elastostatics, Journal of the Mechanics and Physics of Solids, 17, 2, 73-82.

Sahmani S and Aghdam MM (2017). Nonlocal strain gradient shell model for axial buckling and postbuckling analysis of magneto-electro-elastic composite nanoshells. Composites Part B: Engineering.

Seif AE, and Kabir MZ (2015). An efficient analytical model to evaluate the first two local buckling modes of finite cracked plate under tension. Latin American Journal of Solids and Structures 12, 11, 2078-2093.

Seif AE and Kabir MZ (2016). The general form of the elastic stress and displacement fields of the finite cracked plate, Journal of Theoretical and Applied Mechanics 54, 4, 1271-1283.

Seif AE and Kabir MZ (2017). Experimental study on the fracture capacity and fatigue life reduction of the tensioned cracked plate due to the local buckling, Engineering Fracture Mechanics 175, 168-183.

Weertman J, Caracostas CA and Shodja HM (1996). The double slip plane model for the study of short cracks, International Journal of Fatigue, 5(18), 345.

Zarrinzadeh H, Kabir MZ, and Deylami A (2016). Extended finite element fracture analysis of a cracked isotropic shell repaired by composite patch, Fatigue \& Fracture of Engineering Materials \& Structures 39, 11, 1352-1365.

Zarrinzadeh H, Kabir MZ and Deylami A (2017). Crack growth and debonding analysis of an aluminum pipe repaired by composite patch under fatigue loading, Thin-Walled Structures 112, 140-148. 